\title{
Effects of Foam Rolling on Vertical Jump Performance
}

\author{
Andrew Jones \\ Department of Kinesiology, California State University, Fullerton \\ 800 N. State College Blvd., Fullerton, CA 91306 \\ E-mail: jones.andrew11@gmail.com \\ Lee E. Brown (Corresponding author) \\ Department of Kinesiology, California State University, Fullerton \\ 800 N. State College Blvd., Fullerton, CA 91306 \\ E-mail: leebrown@fullerton.edu \\ Jared W. Coburn \\ Department of Kinesiology, California State University, Fullerton \\ 800 N. State College Blvd., Fullerton, CA 91306 \\ Guillermo J. Noffal \\ Department of Kinesiology, California State University, Fullerton \\ 800 N. State College Blvd., Fullerton, CA 91306
}

Received: 05-06- 2015

Accepted: 25-07- 2015

Published: 31-07- 2015
URL: http://dx.doi.org/10.7575/aiac.ijkss.v.3n.3p.38

\begin{abstract}
Background: Foam rolling is a popular activity utilized by strength and conditioning coaches as it is believed to increase muscle length and break up fibrous adhesions located in connective tissue. However, there is little research investigating the effects of foam rolling on athletic performance. Objective: The purpose of this study was to investigate the effects of lower body foam rolling on vertical jump performance. Methods: Twenty males (age $24.05 \pm$ 2.02 years; height $177.43 \pm 6.31 \mathrm{~cm}$; mass $81.41 \pm 8.76 \mathrm{~kg}$ ) volunteered to participate. Subjects completed three days of testing, separated by at least twenty-four hours. Day one consisted of baseline vertical jumps on a force plate, followed by familiarization with foam rolling and control protocols. Subjects returned on days two and three and performed 30second bouts of lower body foam rolling or mimicked foam rolling movements on a skateboard followed by vertical jumps on a force plate. The highest jump from each day was used for statistical analyses. Results: Repeated measures ANOVAs revealed no significant differences in Jump height, impulse, relative ground reaction force, or take-off velocity between conditions. Conclusion: 30 -second bouts of lower body foam rolling do not improve vertical jump performance.
\end{abstract}

Keywords: Dynamic Warm-Up, Foam Rolling, Vertical Jump

\section{Introduction}

Coaches, athletes, and personal trainers commonly use a warm-up prior to competition or physical activity. A traditional warm-up typically consists of submaximal aerobic exercise or static stretching. However, static stretching may be detrimental to sprint and jump performance (Kistler, 2010; Sim, 2009; Vetter, 2007). The research literature has suggested a dynamic warm-up, consisting of exercises that improve dynamic range of motion and simulate specific movements of the sport or activity (Faigenbaum, 2012), is preferential to maximize performance (Aguilar et al., 2012; Carvalho, 2012; Frantz \& Ruiz 2013; Gelen, 2010; Little \& Williams, 2006; McMillian et al., 2006; Pagaduan et al., 2012; Perrier et al., 2011). There are many types of dynamic warm-ups used to increase performance but controlled scientific studies examining critical variables are lacking.

In addition to traditional warm-up methods, massage has been used prior to and after participation to enhance performance and aid recovery (Callaghan, 1993). Similar to static stretching, pre-performance massage may negatively affect performance (Arabaci, 2008; Arazi, 2012; Arroyo-Morales, 2011; Fletcher, 2010). In recent years, foam rolling has become a popular self-massage activity used by clinicians, strength coaches, and personal trainers. Foam rolling is believed to replicate myofascial release, a manual therapy technique that is believed to increase muscle length and break up fibrous adhesions located in the fascia (Barnes, 1997). Recent research has suggested that foam rolling and roller massage devices might improve flexibility (Halperin et al., 2014; MacDonald et al., 2013; MacDonald et al., 2013; Mohr et al., 2014; Roylance et al., 2013; Sullivan et al., 2013). Though its popularity has increased, research investigating its potential impact on performance is limited. Two previous studies have found that foam rolling, using a 
multilevel roller consisting of a polyvinyl chloride core and surrounded by foam, had no effect on performance (Healey, 2013; MacDonald, 2013). Similar to a foam roller, research has investigated a handheld roller massager which uses an individual's upper body to apply force to the muscles (Sullivan, 2013) and found them to be ineffective in improving performance (Mikesky, 2002; Sullivan, 2013). One study found that a handheld roller massage device improved isometric maximum voluntary contraction (MVC) of the plantar flexor muscles when compared to static stretching, but had no effect on electromyography (EMG) (Halperin, 2014).

Many types of pre-participation warm-ups are utilized prior to performance with dynamic and foam rolling being two of the most popular. However, there is limited evidence related to foam rolling. Therefore, the purpose of this study was to investigate the effects of lower body foam rolling on vertical jump performance.

\section{Methods}

\subsection{Participants}

Subjects consisted of twenty recreationally trained males (mean $\pm \mathrm{SD}$; age $24.05 \pm 2.02 \mathrm{yrs}$; ht $177.43 \pm 6.31 \mathrm{~cm}$; mass $81.41 \pm 8.76 \mathrm{~kg}$ ) with no lower body injuries within the past 6 months. All subjects were kinesiology students recruited from classes and received no incentive to participate. They were instructed to refrain from lower body exercise 48 hours before testing. All testing and conditions were performed in a laboratory setting. This study was approved by the University institutional review board and all subjects read and signed an informed consent prior to participation.

\subsection{Overview of the experiment}

Day 1. Upon arrival, subjects were measured for height and mass using a stadiometer (SECA, Ontario, CA, USA) and an electric scale (ES200L, Ohaus, Pine Brook, NJ, USA), respectively. They then completed a dynamic warm-up consisting of high knee pulls, Frankenstein's, and forward gate swings for twenty meters each and were familiarized with the testing protocol (Avila, 2015). Immediately after the dynamic warm-up, they performed three maximal countermovement vertical jumps with arm swing on a force plate. A vertical jump station (EPIC Athletic Performance Inc., Colorado Springs, CO, USA) was used to encourage subjects to jump maximally. Prior to jumping, they were instructed to step on to the force plate, place their hands in front and stand still. When told to jump, they bent their knees and hips while swinging their arms down, and performed a maximal jump, reaching for the vanes of the device. Three jumps were recorded and the highest jump was used for analysis (Avila, 2015). Jump height data from the EPIC vertical jump station was recorded, but not used for data analysis. After completion of the jumps, subjects were familiarized with the foam rolling and control protocols.

Day 2-3. Subjects performed the same dynamic warm-up as day 1. After completion of the dynamic warm-up, they performed the foam rolling protocol or control protocol in a counterbalanced fashion, separated by 48 hours. After completion of the days condition, they immediately performed three countermovement vertical jumps, identical to day 1. All three jumps were recorded and the highest jump was used for analysis.

\subsection{Foam rolling and control protocols}

Foam rolling was performed using a thirty-six inch high-density foam roller (80233-EI-RO, Power Systems, Knoxville, TN, USA). The protocol consisted of one 30-second bout for each of four muscles: gastrocnemii, quadriceps, hamstrings, and glutei, in that order. The right and left sides were foam rolled simultaneously. The control protocol required subjects to perform the same movements as foam rolling, except on a rolling skateboard that replaced the foam roller. Rolling on the skateboard mimicked the foam rolling action without the addition of self-massage that the foam roller provided. They rolled back and forth on the foam roller and skateboard to the beat of a metronome set to 40 beats per minute and were required to switch directions at the sound of each beep, resulting in ten repetitions.

To foam roll the gastrocnemii, subjects sat on the floor with the foam roller underneath them with their hands on the ground to the side and feet in the air. They foam rolled from the top, just below the knee joint, to the bottom, just above the ankle joint, and returned to the top, using their hands to guide the foam roller. For the quadriceps, subjects lied face down on the floor with the foam roller at the top of their quadriceps and their forearms on the ground. They foam rolled from the top to the bottom, just above the patella, and returned to the top, using their forearms to guide the foam roller. For the hamstrings, they sat on the floor with the foam roller underneath them and placed it at the top of the hamstrings with their hands on the ground to the side and feet in the air. They foam rolled from the top of the hamstrings to the bottom, just above the knee joint, and returned to the top, using their hands to guide the foam roller. For the gluteus maximus, they sat on the floor and placed the foam roller at the top of the gluteus maximus with their hands and feet on the ground. They foam rolled from the top of the gluteus maximus to the bottom and returned to the top, using their hands and feet to guide the foam roller.

The control protocol required them to perform the same movements they did while foam rolling, except on a rolling skateboard that replaced the foam roller. To mimic foam rolling of the gastrocnemii and hamstrings, they placed their heels on the middle of the skateboard with their knees fully extended and glutei off the ground then rolled back and forth, using their hands to guide the skateboard. To mimic foam rolling of the quadriceps, they laid prone on the floor and placed their knees on the middle of the skateboard with their feet hanging off the end and in the air then used their forearms and elbows on the ground to roll the skateboard back and forth. To mimic foam rolling of the glutei, they sat on the skateboard with their hands and feet on the ground and used their feet to roll the skateboard back and forth. 
Vertical jumps were performed on an AMTI force plate (Advanced Technology Inc., Watertown, MA, USA), sampling at $1,000 \mathrm{~Hz}$, and interfaced with a computer running custom LabVIEW data collection and analysis software (version 2013, National Instruments Corporation, Austin, TX, USA). Estimated jump height (using the time in the air equation), relative ground reaction force (rGRF), impulse, and take-off velocity (TOV) were recorded (Burnett, 2015).

\subsection{Statistical Analyses}

All values are expressed as mean \pm standard deviation. Four $1 \times 3$ repeated measures ANOVAs analyzed the variables of jump height, rGRF, impulse, and TOV for differences between conditions. Mauchly's test of sphericity was performed for each ANOVA. An a-priori alpha of 0.05 was used to determine statistical significance. All analyses were performed using the Statistical Package for the Social Sciences (SPSS version 20.0).

\section{Results}

Sphericity was not violated for any analysis. There were no significant differences between conditions for any dependent variable.

Table 1. Means \pm SD of vertical jump variables by condition. (rGRF-relative ground reaction force; TOV-take off velocity)

\begin{tabular}{lcccc}
\hline & Baseline & Foam Rolling & Control & p-value \\
\hline Jump Height $(\mathrm{cm})$ & $47.38 \pm 7.26$ & $47.73 \pm 7.22$ & $47.88 \pm 6.78$ & 0.57 \\
Impulse $\left(\mathrm{N}^{*} \mathrm{~s}^{-1}\right)$ & $262.745 \pm 33.50$ & $263.09 \pm 33.82$ & $264.61 \pm 34.32$ & 0.22 \\
rGRF $\left(\mathrm{N}^{*} \mathrm{~kg}^{-1}\right)$ & $24.64 \pm 2.15$ & $24.67 \pm 2.15$ & $24.55 \pm 1.93$ & 0.89 \\
TOV $\left(\mathrm{m}^{*} \mathrm{~s}^{-1}\right)$ & $3.00 \pm .20$ & $3.03 \pm .21$ & $3.03 \pm .217$ & 0.23 \\
\hline
\end{tabular}

\section{Discussion}

In recent years, foam rollers have become popular tools and are believed to increase tissue length and remove fibrous adhesions located in the fascia (Barnes, 1997). To date, there have been few studies investigating the use of foam rollers on performance. The results of this study suggest that the addition of foam rolling after a dynamic warm-up does not acutely improve vertical jump performance. However, baseline vertical jumps, immediately following a dynamic warmup, were not significantly different from foam rolling or control conditions suggesting there was no detrimental effect of foam rolling.

Our study was similar to previous investigations on the effects of foam rolling that have also shown it did not improve performance (Healey, 2013; MacDonald, 2013). These studies used a multilevel roller, rolled different muscle groups and used different durations of time a particular muscle group received treatment but still did not see differences (Healey, 2013; MacDonald, 2013). Handheld roller massage devices were also not shown to improve performance in athletic test performance (Mikesky, 2002) or isometric hamstring performance (Sullivan, 2013). In contrast, Halperin (2014) found that use of a handheld roller massager improved maximal voluntary contraction of the plantar flexors ten minutes after application when compared to static stretching. However, one minute after application of the roller massager or static stretching, there were no significant differences. This was confirmed in our study as performance was not different immediately following the three conditions.

Different durations of foam rolling or handheld roller massage have also been investigated and have demonstrated no increase in performance. Similar to the current study, Healey et al. (2013) foam rolled for 30s per muscle group, while MacDonald et al. (2013) foam rolled the quadriceps for two, 1-minute bouts. These results differ from studies that have investigated the acute effect of massage on performance where five to six minutes of lower body massage negatively affected performance (Arabaci, 2008; Arazi, 2012; Arroyo-Morales, 2011). Additionally, one minute of massage to the gastrocnemius, hamstrings, gluteals, and quadriceps and thirty seconds to the tibialis anterior also negatively affected 20-meter sprint performance (Fletcher, 2010). Arroyo-Morales (2011) concluded that the negative effects of massage on isokinetic knee extensor performance could be due to an increase in parasympathetic nervous system activity.

The sequence of warm-up activities used in the present study did not increase vertical jump performance, but it did not negatively affect it either. We had subjects perform a standardized dynamic warm-up prior to foam rolling or control conditions, then immediately vertical jump. Similarly, Healey (2013) had subjects perform a standardized dynamic warm-up prior to foam rolling and planking conditions while MacDonald (2013) had subjects perform a five minute cycle ergometer warm-up prior to foam rolling and control conditions. Previous studies investigating pre-performance massage have found that it negatively affected performance (Arabaci, 2008; Arazi, 2012; Arroyo-Morales, 2011; Fletcher, 2010). Perhaps performing a warm-up after massage could minimize the negative effects (Fletcher, 2010; Goodwin, 2007). Other possible limitations of the current study were the type of foam rollers used and the duration of rolling. Since vertical jump performance was not hampered by the addition of foam rolling, further manipulation of these factors may lead to jump increases. 
Though it was not the aim of this study and was not measured, foam rolling and handheld roller massage devices have been shown to increase flexibility (Halperin, 2014; MacDonald, 2013; Mohr, 2014; Roylance, 2013; Sullivan, 2013). As little as five seconds of application of a handheld massage device increased flexibility of the hamstrings (Sullivan, 2013). Also, adding foam rolling to a static stretching or postural alignment program significantly increased flexibility in subjects who had less than average flexibility (Roylance, 2013) while foam rolling and static stretching also increased flexibility in subjects with less than 90 degrees hip flexion range of motion (Mohr, 2014). Therefore, foam rolling may result in changes in flexibility that are unrelated to performance.

\section{Conclusion}

The results of this study indicate that the addition of foam rolling after a dynamic warm-up does not increase acute vertical jump performance. Given the relatively small amount of data on foam rolling's effects on performance, future investigations should consider different types of rollers and materials. Furthermore, longer rolling durations and different performance measures should be studied.

\section{References}

Aguilar A.J., DiStefano L.J., Brown C.N., Herman D.C., Guskiewicz K.M., Padua A.P. (2012). A dynamic warm-up increases quadriceps strength and hamstring flexibility. Journal of Strength and Conditioning Research, 26(4), 11301141.

Arabaci R. (2008). Acute effects of pre-event lower limb massage on explosive and high-speed motor capacities and flexibility. Journal of Sports Science and Medicine, 7, 549-555.

Arazi H., Asadi A., Hoseini K. (2012). Comparison of two different warm-ups (static stretching and massage) effects on flexibility and explosive power. Acta Kinesiologica, 6(1), 55-59.

Arroyo-Morales M., Fernandez-Lao C., Ariza-Garcia A., Toro-Velasco, I., Winters, M., Diaz-Rodriguez, L., CantareroVillanueva, I., Huljbregts, P., Fernandez-De-Las-Penas, C. (2011). Psychophysiological effects of preperformance massage before isokinetic exercise. Journal of Strength and Conditioning Research, 25(2), 481-488.

Avila B.J., Brown L.E., Coburn J.W., Statler T.A. (2015). Effects of imagery on force production and jump performance. Journal of Exercise Physiology Online, 18(4), 42-48.

Barnes M.F. (1997). The basic science of myofascial release: morphologic change in connective tissue. Journal of Bodywork and Movement Therapies, 1(4), 231-238.

Burnett K.A., Brown L.E., Kersey R.D., Tsang K.W. (2015). Effect of ankle bracing vs. taping on vertical jump performance. Journal of Athletic Enhancement, 4(1).

Callaghan M.J. (1993). The role of massage in the management of the athlete: a review. British Journal of Sports Medicine, 27, 28-33.

Carvalho F.L.P., Carvalho M.C.G.A., Simao R., Gomes, T.M., Costa, P.B., Neto, L.B., Carvalho, R.L.P., Dantas, E.H.M. (2012). Acute effects of a warm-up including active, passive, and dynamic stretching on vertical jump performance. Journal of Strength and Conditioning Research, 26(9), 2447-2452.

Faigenbaum A.D. (2012). NSCA's Guide to Program Design. Illinois: Human Kinetics, (Chapter 3).

Fletcher I.M. (2010). The effects of precompetition massage on kinematic parameters of 20-m sprint performance. Journal of Strength and Conditioning Research, 24(5), 1179-1183.

Frantz T.L., Ruiz M.D. (2011). Effects of dynamic warm-up on lower body explosiveness among collegiate baseball players. Journal of Strength and Conditioning Research, 25(11), 2985-2990.

Gelen E. (2010). Acute effects of different warm-up methods on sprint, slalom dribbling, and penalty kick performance in soccer players. Journal of Strength and Conditioning Research, 24(4), 950-956.

Goodwin J.E., Glaister M., Howatson G., Lockey R.A., McInnes G. (2007). Effect of preperformance lower-limb massage on thirty-meter sprint running. Journal of Strength and Conditioning Research, 21(4), 1028-1031.

Halperin I., Aboodarda S.J., Button D.C., Andersen L.L., Behm D.G. (2014). Roller massage improves range of motion of plantar flexor muscles without subsequent decreases in force parameters. The International Journal of Sports Physical Therapy, 9(1), 92-102.

Healey K.C., Hatfield D.L., Blanpied P., Dorfman L.R., Riebe D. (2013). The effects of myofascial release with foam rolling on performance. Journal of Strength and Conditioning Research, 28(1), 61-68.

Kistler B.M., Walsh M.S., Horn T.S., Cox R.H. (2010). The acute effects of static stretching on the sprint performance of collegiate men in the 60- and 100-meter dash after a dynamic warm-up. Journal of Strength and Conditioning Research, 24(9), 2280-2284.

Little T., Williams A.G. (2006). Effects of differential stretching protocols during warm-ups in professional soccer players. Journal of Strength and Conditioning Research, 20(1), 203-207. 
MacDonald G.Z., Penney M.D.H., Mullaley M.E., Cuconato, A.L., Drake, C.D.J., Behm, D.G., Button, D.C. (2013). An acute bout of self-myofascial release increases range of motion without a subsequent decrease in muscle activation or force. Journal of Strength and Conditioning Research, 27(3), 812-821.

MacDonald G.Z., Button D.C., Drinkwater E.J., Behm D.G. (2014). Foam rolling as a recovery tool following an intense bout of physical activity. Medicine and Science in Sports and Exercise, 46(1), 131-142.

Mikesky A.E., Bahamonde R.E., Stanton K., Alvey T., Fitton T. (2002). Acute effects of the stick on strength, power, and flexibility. Journal of Strength and Conditioning Research, 16(3), 446-450.

Mohr A.R., Long B.C., Goad C.L. (2013). Foam rolling and static stretching on passive hip flexion range of motion. Journal of Sport Rehabilitation, 23, 296-299.

Pagaduan J.C., Pojskic H., Uzicanin E., Babajic F. (2012). Effect of various warm-up protocols on jump performance in college football players. Journal of Human Kinetics, 35, 127-132.

Perrier E.T., Pavol M.J., Hoffman M.A. (2011). The acute effects of a warm-up including static or dynamic stretching on countermovement jump height, reaction time, and flexibility. Journal of Strength and Conditioning Research, 25(7), 1925-1931.

Roylance D.S., George J.D., Hammer A.M., Rencher, N., Gellingham, G.W., Hager, R.L., Myrer, W.J. (2013). Evaluating acute changes in joint range-of-motion using self-myofascial release, postural alignment exercises, and static stretches. International Journal of Exercise Science, 6(4), 310-319.

Sim A.Y., Dawson B.T., Guelfi K.J., Wallman K.E., Young W.B. (2009). Effects of static stretching on repeated sprint performance. Journal of Strength and Conditioning Research, 23(7): 2155-2162.

Sullivan K.M., Silvey D.B.J., Button D.C., Behm D.G. (2013). Roller-massage application to the hamstrings increases sit-and-reach range of motion within five to ten seconds without performance impairments. The International Journal of Sports Physical Therapy, 8(3), 228-236.

Vetter R.E. (2007). Effects of six warm-up protocols on sprint and jump performance. Journal of Strength and Conditioning Research, 21(3), 819-823. 